OPEN ACCESS

Edited by:

Gary Tse,

Second Hospital of Tianjin Medical

University, China

Reviewed by:

Christien Li.

NHS England, United Kingdom

Xintao Li

Dalian Medical University, China

*Correspondence:

Ji Zhang

doctorzhangji@163.com

Yidong Wei

ywei@tongji.edu.cn

tThese authors have contributed equally to this work and share first authorship

Specialty section:

This article was submitted to

Cardiac Rhythmology,

a section of the journal

Frontiers in Cardiovascular Medicine

Received: 08 March 2021

Accepted: 23 August 2021

Published: 22 September 2021

Citation:

Luo J, Liu B, Li H, Xu S, Gong M, Li Z,

Qin X, Shi B, Hao C, Zhang J and Wei $Y$ (2021) Prognostic Impact of the

Symptom of New-Onset Atrial

Fibrillation in Acute Myocardial

Infarction: Insights From the

NOAFCAMI-SH Registry.

Front. Cardiovasc. Med. 8:677695.

doi: 10.3389/fcvm.2021.677695

\section{Prognostic Impact of the Symptom of New-Onset Atrial Fibrillation in Acute Myocardial Infarction: Insights From the NOAFCAMI-SH Registry}

\author{
Jiachen Luo ${ }^{\dagger}$, Baoxin $\mathrm{Liu}^{\dagger}$, Hongqiang $\mathrm{Li}^{\dagger}$, Siling Xu, Mengmeng Gong, Zhiqiang Li, \\ Xiaoming Qin, Beibei Shi, Chuanzhen Hao, Ji Zhang ${ }^{*}$ and Yidong Wei ${ }^{*}$
}

Department of Cardiology, Shanghai Tenth People's Hospital, Tongji University School of Medicine, Shanghai, China

Background: New-onset atrial fibrillation (NOAF) is a common complication during acute myocardial infarction (AMI) and sometimes can be completely asymptomatic, but the clinical implications of these asymptomatic episodes require further characterization. The objective of this study was to investigate the short- and long-term prognostic impact of post-MI NOAF based on the presence of AF-related symptoms.

Methods: The New-Onset Atrial Fibrillation Complicating Acute Myocardial Infarction in ShangHai (NOAFCAMI-SH) registry was a retrospective cohort including participants with AMI without a documented history of AF. Patients with NOAF were divided into two groups according to the AF-related symptoms. The primary endpoint was all-cause mortality.

Results: Of 2,399 patients included, 278 (11.6\%) developed NOAF of whom $145(6.0 \%)$ with asymptomatic episodes and 133 (5.5\%) with symptomatic ones. During hospitalization, 148 patients died [106, 10, and 32 in the sinus rhythm (SR), asymptomatic, and symptomatic NOAF groups, respectively]. After multivariable adjustment, only symptomatic NOAF was associated with in-hospital mortality [odds ratio (OR): 2.32, 95\% confidence interval $(\mathrm{Cl})$ : 1.36-3.94] compared with SR. Over a median follow-up of 2.7 years, all-cause mortality was 3.2, 12.4, and $11.8 \%$ per year in the SR, asymptomatic, and symptomatic NOAF groups, respectively. After adjustment for confounders, it was the asymptomatic NOAF [hazard ratio $(\mathrm{HR}): 1.61,95 \% \mathrm{Cl}: 1.09-2.37)$ rather than the symptomatic one (HR: 1.37, 95\% Cl: 0.88-2.12) that was significantly related to mortality. Similar results were also observed for cardiovascular mortality [HRs and 95\% Cl were 1.71 (1.10-2.67) and $1.25(0.74-2.11)$ for asymptomatic and symptomatic NOAF, respectively]. Both asymptomatic and symptomatic NOAF episodes were associated with heart failure, whereas only those with symptomatic NOAF were at heightened risk of ischemic stroke. Our exploratory analysis further identified patients with asymptomatic high-burden NOAF as the highest-risk population (mortality: 19.6\% per year). 
Conclusion: Among patients with AMI, symptomatic NOAF is related to in-hospital mortality and asymptomatic NOAF is associated with poor long-term survival.

Registration: URL: https://clinicaltrials.gov/; Unique identifier: NCT03533543.

Keywords: acute myocardial infarction, atrial fibrillation, symptom, mortality, heart failure, ischemic stroke

\section{INTRODUCTION}

Atrial fibrillation (AF) is one of the most common arrhythmias worldwide, with a growing public burden due to the aging of the population. Atrial fibrillation is often intermittent and asymptomatic; sometimes it can only be detected during the diagnostic evaluation of patients presenting with cryptogenic stroke $(1,2)$. Debates over the screening modality, prognostic impact, and management of these asymptomatic AF episodes still exist (3-10).

Among patients with acute myocardial infarction (AMI), nearly $5-20 \%$ of whom will develop new-onset atrial fibrillation (NOAF), which is generally accompanied by increased risks of subsequent death and ischemic stroke $(11,12)$. Similar to the condition in the general population, NOAF during AMI can also be completely asymptomatic (13). Given the potential adverse impact of asymptomatic AF, researches focusing on the prevalence, clinical profiles, as well as prognostic implications of asymptomatic NOAF complicating AMI are of great clinical importance in helping the decision-making for out-patient ECG monitoring strategy as well as stroke and decompensated heart failure (HF) prophylaxis (14). However, until now, only in the sensitivity analysis of an observational AMI registry had researchers explored the impact of asymptomatic AF on prognosis (15).

Accordingly, using data from the New-Onset Atrial Fibrillation Complicating Acute Myocardial Infarction in ShangHai (NOAFCAMI-SH) registry, we aimed to perform a retrospective analysis to describe the clinical features and to investigate the impact of asymptomatic and symptomatic NOAF during AMI on in-hospital and long-term survival.

\section{METHODS}

\section{Study Population}

The design of the NOAFCAMI-SH registry has been previously described $(16,17)$. In brief, this is a retrospective cohort study from a tertiary academic medical center, which included patients who experienced an AMI, did not have a medical history of $\mathrm{AF}$, and received continuous electronic monitoring (CEM) during hospitalization between February 2014 and March 2018. For the present analysis, all NOAFCAMI-SH participants were included, while event-free survival was only analyzed among individuals who were discharged alive with morbidity followup available. Supplementary Figure 1 illustrates a CONSORT diagram of the study population. This study was conducted according to the Declaration of Helsinki, and the protocol of the NOAFCAMI-SH registry had been approved by the ethics committee of the Shanghai Tenth People's Hospital. Informed consent was not required as all data were deidentified during the analytic stages.

\section{Asymptomatic and Symptomatic NOAF Ascertainment}

The occurrence of AF episodes was identified according to the individuals' CEM data. AF was diagnosed based on the consensus guidelines as follows: absolutely irregular RR intervals, no distinct $\mathrm{P}$ waves, and lasted for at least $30 \mathrm{~s}$ (14). NOAF was defined as patients without a history of AF who developed the first documented AF during the index AMI hospitalization. Patients would be systematically interviewed for their symptoms whenever an AF episode presented. Symptomatic NOAF was determined if the occurrence of NOAF event was simultaneously accompanied by any discomfort (e.g., chest tightness, palpitation, shortness of breath, etc.) or the need for emergent cardioversion. Asymptomatic NOAF was determined as any asymptomatic events of NOAF (13). The analyzed population was divided into three groups: sinus rhythm (SR), asymptomatic NOAF, and symptomatic NOAF.

\section{Baseline Covariates}

Baseline covariates consisted of patient demographics, medical history, in-hospital examination, and medications, which were ascertained by a detailed review of electronic medical records during or before the index hospitalization. Demographics included age, sex, smoking status, and body mass index. Medical history included hypertension, diabetes, hyperlipidemia, chronic kidney disease (CKD), HF, MI, percutaneous coronary intervention (PCI), peripheral artery disease (PAD), and stroke/transient ischemic attack (TIA). The in-hospital examination included creatinine, peak-TnT, peak NT-pro BNP, and angiographic and echocardiographic data. Medications included the use of antiplatelet agents, oral anticoagulants, ACE inhibitor/angiotensin receptor blocker (ACEI/ARB), $\beta$-blocker, diuretic, and amiodarone.

\section{Outcome Measures}

The primary outcome was all-cause death. Secondary outcomes included cardiovascular death, HF hospitalization, and ischemic stroke. All deaths without a definite non-cardiovascular cause (e.g., severe pneumonia, malignant tumors, end-stage renal disease, traffic accidents, etc.) would be treated as cardiovascular deaths. HF hospitalization was defined as any admission with a primary diagnosis of $\mathrm{HF}$ at discharge requiring intravenous diuretics. Ischemic stroke was defined as the occurrence of a new focal neurologic deficit considered to be ischemic in origin, with signs or symptoms lasting over $24 \mathrm{~h}$. Patients were followed from the index discharge to the date of the presence of an outcome 
of interest, death, or last follow-up (April 2019), whichever came first. Clinical outcomes were evaluated by a comprehensive review of the patient's medical records.

\section{Statistical Analysis}

Categorical variables were presented as frequencies and proportions and were compared with the $\chi^{2}$ or Fisher's exact test, as appropriate. Continuous variables were presented as means or medians and were compared with the one-way analysis of variance or Kruskal-Wallis test, as appropriate.

Treating SR as the reference, multivariable logistic regression models were established to investigate the association of asymptomatic and symptomatic NOAF with in-hospital death. Odds ratios (ORs) and 95\% confidence intervals (CIs) were calculated using three multivariable logistic regression models. In model 1, we adjusted for age and sex. In model 2, we

TABLE 1 | Patient characteristics.

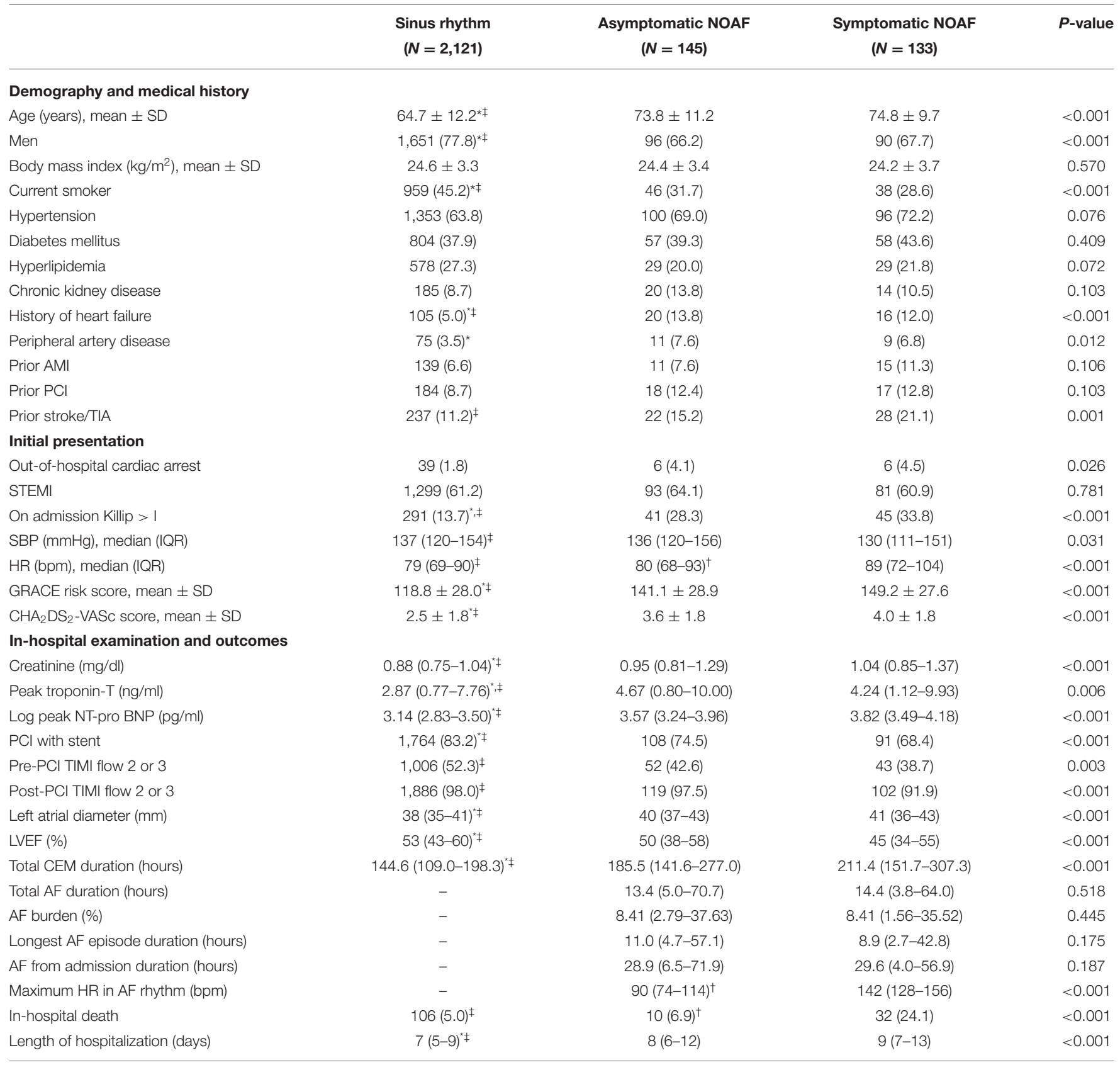

Values presented as mean $\pm S D$, median (IQR), or $n$ (\%). The level of statistical significance was $p<0.017$ for ${ }^{*}$ sinus rhythm vs. asymptomatic NOAF; ${ }^{\dagger}$ asymptomatic NOAF vs. symptomatic NOAF; and $\ddagger$ sinus rhythm vs. symptomatic NOAF, after multiple comparisons. AMI, acute myocardial infarction; CEM, continuous electronic monitoring; HR, heart rate;

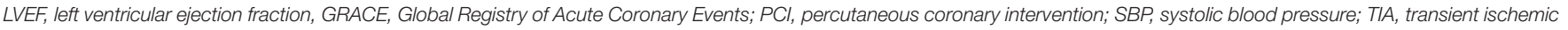
attack; TIMI, thrombolysis in myocardial infarction. 
further adjusted for cardiovascular risk factors (current smoker, hypertension, diabetes, CKD, previous MI, previous stroke/TIA, and PAD). In model 3, we further adjusted for admission characteristics [systolic blood pressure (SBP), heart rate, initial Killip class, out-of-hospital cardiac arrest, and left ventricular ejection fraction (LVEF)] and in-hospital PCI. For long-term survival analyses, event-free survival curves were estimated with the Kaplan-Meier method and compared using log-rank tests. We calculated hazard ratios (HRs) with $95 \%$ CIs by multivariable Cox proportional hazards analyses, and the candidate covariates included the following: (i) the Global Registry Acute Coronary Events (GRACE) risk score as a whole and (ii) variables in model 3. Of note, for the ischemic stroke evaluation, the individual components (age, sex, a history of HF, hypertension, diabetes, stroke/TIA, and vascular disease) in the $\mathrm{CHA}_{2} \mathrm{DS}_{2}$-VASc score were adjusted. The assumption of proportional hazards was verified by a visual examination of the $\log$ (minus log) curves.
The propensity score method was also used to compare the SR with either asymptomatic or symptomatic NOAF. Binary logistic regression analysis was used to calculate propensity scores to balance baseline characteristics (covariates listed in Supplementary Material). Two sets of propensity scores were calculated, one for comparing SR with asymptomatic NOAF and the other to compare SR with symptomatic NOAF. Matching was performed with a 1:3 matching protocol without replacement, using a caliper width equal to 0.10 of the SD of the propensity score.

\section{Subgroup and Sensitivity Analyses}

The associations between asymptomatic and symptomatic NOAF and mortality were explored in subgroups as follows: age ( $\geq 75$ vs. $<75$ years), gender (male vs. female), AMI type (STEMI vs. NSTEMI), and whether the patient underwent PCI (yes vs. no). Additionally, several sensitivity analyses were

TABLE 2 | Medications during hospitalization and at discharge.

\begin{tabular}{|c|c|c|c|c|}
\hline & $\begin{array}{c}\text { Sinus rhythm } \\
(N=2,121)\end{array}$ & $\begin{array}{l}\text { Asymptomatic NOAF } \\
\qquad(N=145)\end{array}$ & $\begin{array}{l}\text { Symptomatic NOAF } \\
\qquad(N=133)\end{array}$ & $P$-value \\
\hline \multicolumn{5}{|c|}{ Medications during hospitalization } \\
\hline $\mathrm{P}_{2} \mathrm{Y}_{12}$ receptor inhibitor & $2,086(98.3)$ & $140(96.6)$ & $132(99.2)$ & 0.185 \\
\hline GP $\left\|_{\mathrm{b}} /\right\|_{\mathrm{a}}$ inhibitor & $1,803(85.0)$ & $123(84.8)$ & $107(80.5)$ & 0.366 \\
\hline ACEI/ARB & $1,326(62.5)$ & $1,326(62.5)$ & $86(64.7)$ & 0.876 \\
\hline$\beta$-blocker & $1,626(76.7)$ & $100(69.0)$ & $103(77.4)$ & 0.103 \\
\hline Statin & $2,073(97.7)$ & $139(95.9)$ & $129(97.0)$ & 0.328 \\
\hline Diuretic & $665(31.4)^{\star} \ddagger$ & $95(65.5)^{\dagger}$ & $110(82.7)$ & $<0.001$ \\
\hline Amiodarone & $237(11.2)^{\star} \ddagger$ & $67(46.2)^{\dagger}$ & $118(88.7)$ & $<0.001$ \\
\hline ACEI/ARB & $1,205(59.8)$ & $73(54.1)$ & $54(53.5)$ & 0.208 \\
\hline$\beta$-blocker & $1,488(73.8)^{\star}$ & $77(57.0)$ & $64(63.4)$ & $<0.001$ \\
\hline Statin & $1,939(96.2)^{*}$ & $123(91.1)$ & $96(95.0)$ & 0.014 \\
\hline Diuretic & $258(12.8)^{\star} \ddagger$ & $37(27.4)$ & $31(30.7)$ & $<0.001$ \\
\hline Amiodarone & $25(1.2)^{\star} \neq$ & $11(8.1)^{\dagger}$ & $21(20.8)$ & $<0.001$ \\
\hline
\end{tabular}

Values presented as $n(\%)$. The level of statistical significance was $p<0.017$ for ${ }^{*}$ sinus rhythm vs. asymptomatic NOAF; ${ }^{\dagger}$ asymptomatic NOAF vs. symptomatic NOAF; and ${ }^{\ddagger}$ sinus rhythm vs. symptomatic NOAF, after multiple comparisons. ACEI/ARB, angiotensin-converting enzyme inhibitors/angiotensin receptor blocker.

TABLE 3 | Unadjusted and multivariable-adjusted logistic models for in-hospital mortality.

\begin{tabular}{|c|c|c|c|c|c|c|c|c|}
\hline & Unadjusted & $P$-value & Model 1 & $P$-value & Model 2 & $P$-value & Model 3 & $P$-value \\
\hline & OR (95\% Cl) & & OR (95\% Cl) & & OR $(95 \% \mathrm{Cl})$ & & OR (95\% Cl) & \\
\hline Sinus rhythm & Reference & - & Reference & - & Reference & - & Reference & - \\
\hline Asymptomatic NOAF & $1.41(0.72-2.76)$ & 0.318 & 0.79 (0.39-1.57) & 0.500 & $0.85(0.42-1.70)$ & 0.642 & $0.53(0.24-1.17)$ & 0.117 \\
\hline Symptomatic NOAF & 6.02 (3.87-9.38 & $<0.001$ & 3.59 (2.26-5.70) & $<0.001$ & $3.59(2.24-5.74)$ & $<0.001$ & $2.32(1.36-3.94)$ & 0.002 \\
\hline
\end{tabular}

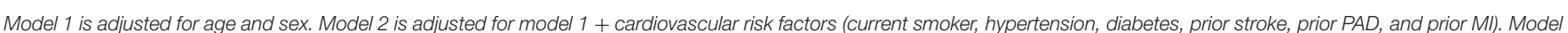

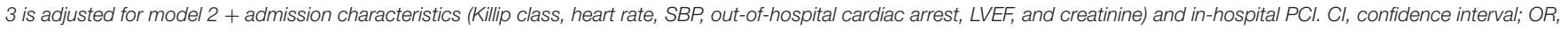
odds ratio; PAD, peripheral artery disease. Other variables refer to Table 1. 
also conducted. First, adjustment for differences in baseline characteristics was performed using stabilized inverse probability of treatment weighting (IPTW) models. Second, we further adjusted for medication usage (aspirin, ACEI/ARB, $\beta$-blocker, statin, and oral anticoagulant). Third, we censored patients who died within 1 month after discharge. Fourth, to minimize the potential misclassification of NOAF, we repeated the analysis by excluding those with prior stroke/TIA who were at high risk of asymptomatic $\mathrm{AF}(1,2)$. Moreover, we performed an exploratory analysis in which patients with or without AF symptoms were further grouped according to the NOAF burden of $10.87 \%$ to investigate its interaction effects with AF symptoms, given the prognostic importance of the burden of post-MI NOAF (17). All analyses were performed with Stata v14.0 and R v3.6.3. A two-sided $P<0.05$ was thought to be statistically significant.

\section{RESULTS}

\section{Study Population}

Among the 2,399 participants included in the NOAFCAMI$\mathrm{SH}$ registry, 278 (11.6\%) developed NOAF during their hospital stay. Among those, 145 (6.0\%) experienced asymptomatic NOAF and $133(5.5 \%)$ had symptomatic NOAF. Baseline characteristics are shown in Table 1. Patients in the NOAF group were older, mainly female, more likely to have a history of $\mathrm{HF}$, with a higher GRACE score and $\mathrm{CHA}_{2} \mathrm{DS}_{2}$-VASc score, with a lower LVEF value, and less likely to undergo PCI for reperfusion when compared with those in the SR group. No significant difference was observed between asymptomatic and symptomatic NOAF except admission heart rate. Table 2 demonstrates the use of medications. Patients with symptomatic NOAF were more likely to be prescribed vasoactive agents, diuretics, and amiodarone when compared with the other two groups.

\section{In-hospital Mortality}

A total of $148(6.2 \%)$ patients died during hospitalization, of whom $106(5.0 \%), 10(6.9 \%)$, and $32(24.1 \%)$ were in the $\mathrm{SR}$, asymptomatic NOAF, and symptomatic NOAF groups, respectively. As shown in Table 3, when treating the SR as the reference, the fully adjusted ORs and 95\% CIs were 0.60 $(0.28-1.29)$ and $2.35(1.38-3.98)$ for the asymptomatic and symptomatic NOAF, respectively.

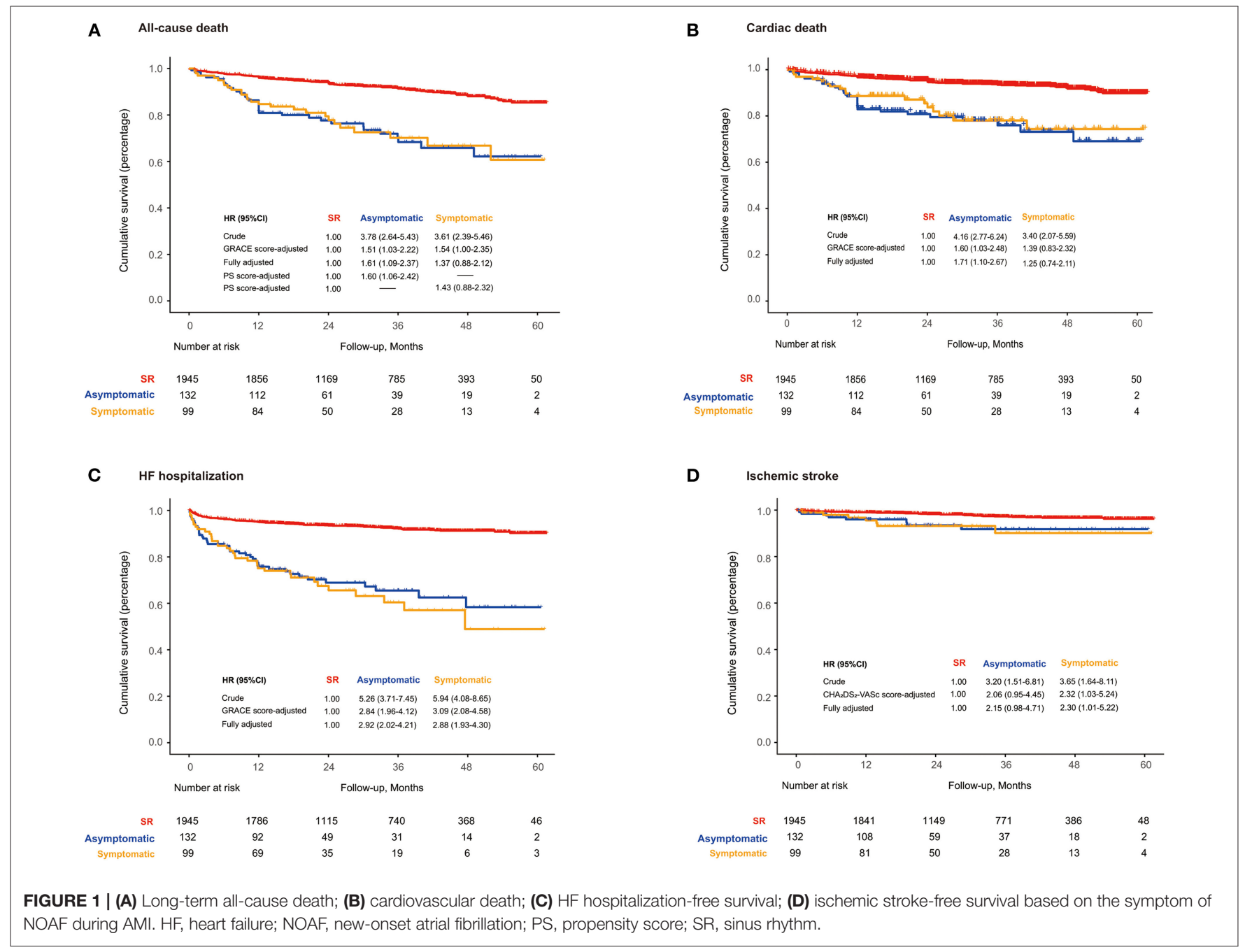




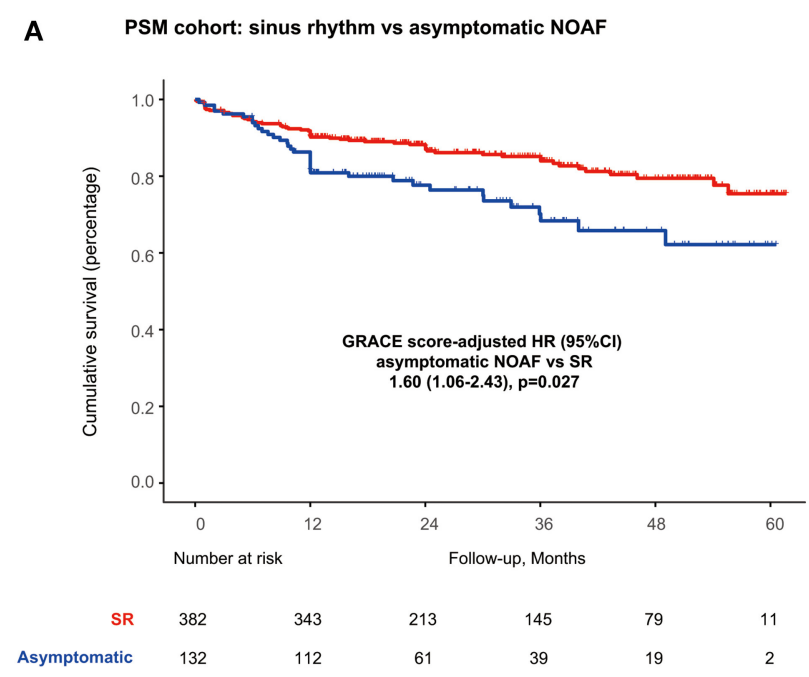

C
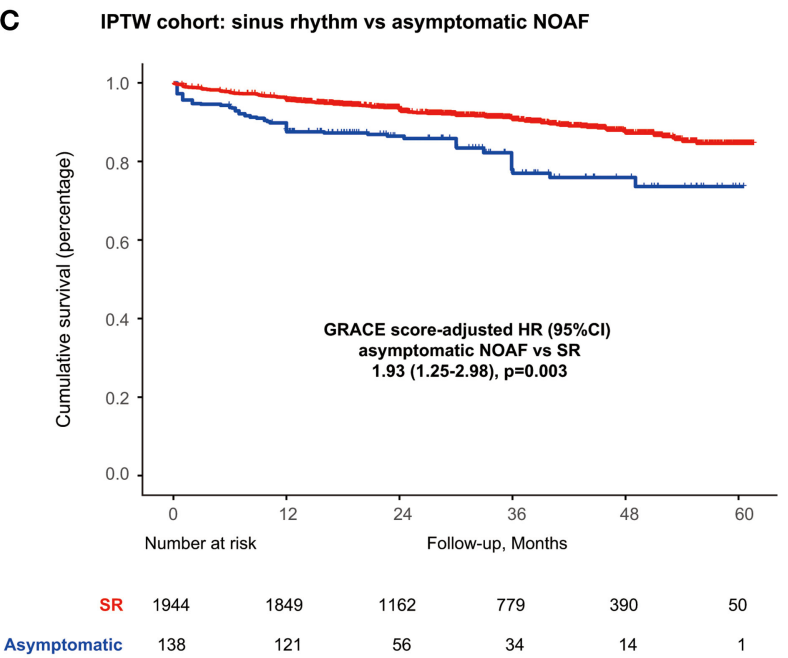

B PSM cohort: sinus rhythm vs symptomatic NOAF

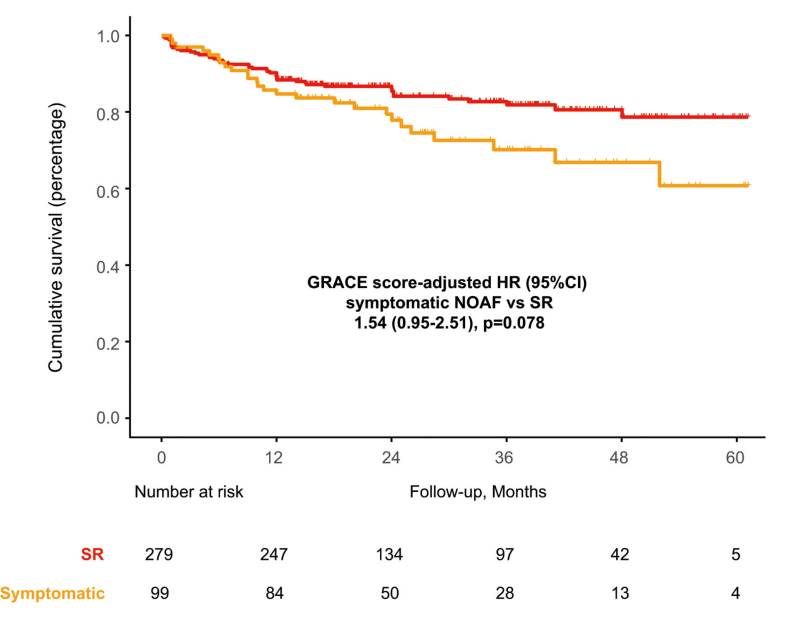

D IPTW cohort: sinus rhythm vs symptomatic NOAF

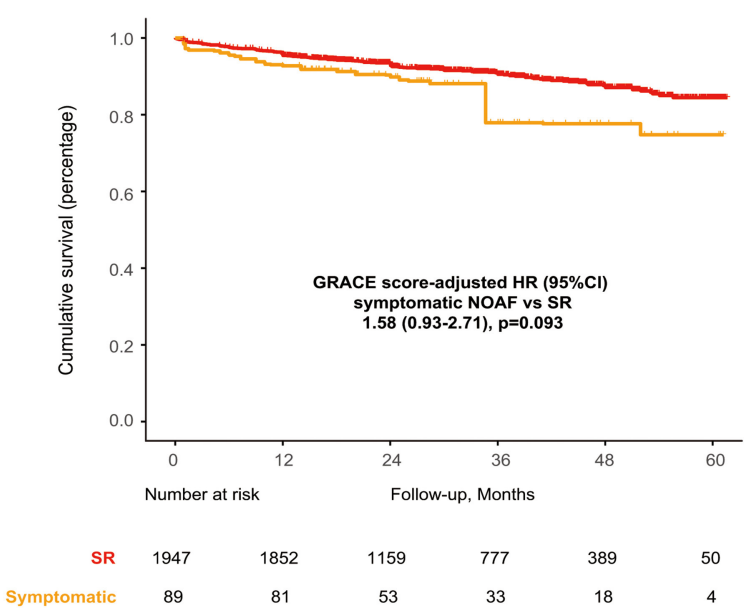

FIGURE 2 | Long-term survival in PSM cohorts for (A) SR vs. asymptomatic NOAF and (B) SR vs. symptomatic NOAF and in IPTW cohorts for (C) SR vs. asymptomatic NOAF and (D) SR vs. symptomatic NOAF. IPTW, inverse probability of treatment weighting; PSM, propensity score matching.

\section{Long-Term Outcomes}

Over a median follow-up of 2.7 years (IQR: 1.6-3.9), all-cause mortality was $3.2 \%(2.7-3.7 \%)$ for the SR, 12.4 (8.9-17.2\%) for asymptomatic NOAF, and $11.8 \%(8.0-17.3 \%)$ for symptomatic NOAF. When compared with SR, the HRs and 95\% CIs were $1.51(1.03-2.22)$ for asymptomatic NOAF and $1.54(1.00-2.35)$ for symptomatic NOAF after accounting for GRACE score and $1.61(1.09-2.37)$ and $1.37(0.88-2.12)$, respectively, after full adjustment. After adjustment for the propensity scores, the HR for asymptomatic NOAF was $1.60(1.06-2.42)$ and $1.43(0.88-$ 2.32) for symptomatic NOAF, compared with the SR (Figure 1A). Moreover, it was the asymptomatic NOAF (HR: 1.71, 95\% CI: 1.10-2.67) rather than the symptomatic one (HR: 1.25, 95\% CI: 0.74-2.11) that was significantly associated with elevated cardiovascular mortality (Figure 1B). Both asymptomatic (HR:
2.92, 95\% CI: 2.02-4.21) and symptomatic NOAF (HR: 2.88, 95\% CI: 1.93-4.30) episodes were significantly associated with increased risk of HF hospitalization (Figure 1C). Only patients with symptomatic NOAF were at heightened long-term risk of ischemic stroke compared to those with SR (HR: 2.30, 95\% CI: 1.01-5.22; Figure 1D).

As illustrated in Supplementary Figure 2, patient's characteristics were well-balanced in the propensity scorematched (PSM) cohorts. In the matched cohorts, long-term mortality was $6.3 \%$ (4.9-8.1\%) for the SR and $12.4 \%(8.9-17.2 \%)$ for asymptomatic NOAF (asymptomatic NOAF vs. SR, HR: 1.60, 95\% CI: $1.06-2.43 ; P=0.027)$ and $7.0 \%(5.2-9.4 \%)$ for the SR and $11.8 \%(8.0-17.3 \%)$ for symptomatic NOAF (symptomatic NOAF vs. SR, HR: 1.58, 95\% CI: 0.93-2.71; $P=0.093$ ) (Figures 2A,B). IPTW analyses demonstrated similar results (Figures 2C,D). 


\section{Heart Rhythm}

HR $(95 \% \mathrm{Cl}) \quad P$-value $\quad P_{\text {interaction }}$

\section{All-cause mortality \\ Age}

Age $\geq 75$

Age $<75$

Sex

Male

Female

MI type

STEMI

NSTEMI

$\mathrm{PCl}$ with stent

Yes

No
Sinus rhythm Silent NOAF Symptomatic NOAF

Sinus rhythm Silent NOAF Symptomatic NOAF

Sinus rhythm Silent NOAF Symptomatic NOAF Sinus rhythm Silent NOAF Symptomatic NOAF

Sinus rhythm Silent NOAF Symptomatic NOAF Sinus rhythm Silent NOAF Symptomatic NOAF

Sinus rhythm Silent NOAF Symptomatic NOAF Sinus rhythm Silent NOAF Symptomatic NOAF
Ref

$1.55(0.99,2.42)$

$1.36(0.80,2.30)$

Ref.

$2.39(1.09,5.24) \quad 0.030$

$1.39(0.58,3.36) \quad 0.458$

Ref.

$1.78(1.09,2.89)$

$1.29(0.76,2.19)$

Ref.

$1.39(0.73,2.64) \quad 0.315$

$1.40(0.63,3.14) \quad 0.408$

Ref.

$1.50(0.84,2.67)$

0.166

0.483

Ref.

$1.92(1.10,3.35)$

$1.57(0.86,2.87)$

0.022

0.138

Ref.

$1.63(0.95,2.79)$

$1.53(0.86,2.73)$

Ref.

$1.52(0.84,2.76)$

$1.35(0.65,2.79)$ $\overline{0.075}$

0.147

0.168

0.419
0.809

0.983

0.691

0.834

0.021

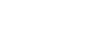

(1)

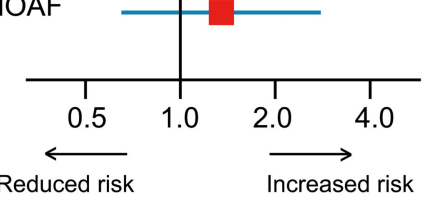

FIGURE 3 | Subgroup analysis. MI, myocardial infarction; PCl, percutaneous coronary intervention.

No significant heterogeneity in HR was observed across all subgroups (Figure 3). In the sensitivity analysis, results remained robust after adjustment for the medication usage, censoring patients who died within 1 month after discharge or excluding those with a prior stroke/TIA (Supplementary Figure 3). In the exploratory analysis, patients with asymptomatic high-burden NOAF were identified as the highest-risk population with allcause mortality of $19.6 \%$ per year [fully adjusted HR (treating SR as the reference): $1.81,95 \% \mathrm{CI}: 1.10-2.99, P=0.020$; Figure 4 ).

\section{DISCUSSION}

The present analysis found that the incidence rate of postMI asymptomatic NOAF was 6.0\%. Symptomatic NOAF episodes were significantly associated with increased inhospital mortality, whereas only asymptomatic episodes were related to poor long-term survival. Similar results were observed for the risk evaluation of cardiovascular death. Notably, patients with high-burden asymptomatic NOAF represented the highest-risk population for allcause death. In addition, when compared to the SR, both asymptomatic and symptomatic NOAF were associated with a heightened risk of HF hospitalization, but only symptomatic NOAF was challenged by a higher risk of ischemic stroke.

AF represents the most common arrhythmia in daily clinical practice, but its incidence is still thought to be underestimated since AF sometimes can be completely asymptomatic. Nowadays, technical advances in cardiac implantable electronic devices allow for the early detection of asymptomatic AF, and the prevalence of asymptomatic AF is varied across different settings (18), but data about asymptomatic AF in the AMI population remain limited. In our study, the incidence rate of in-hospital asymptomatic NOAF was $\approx 6.0 \%$, which is lower than that 


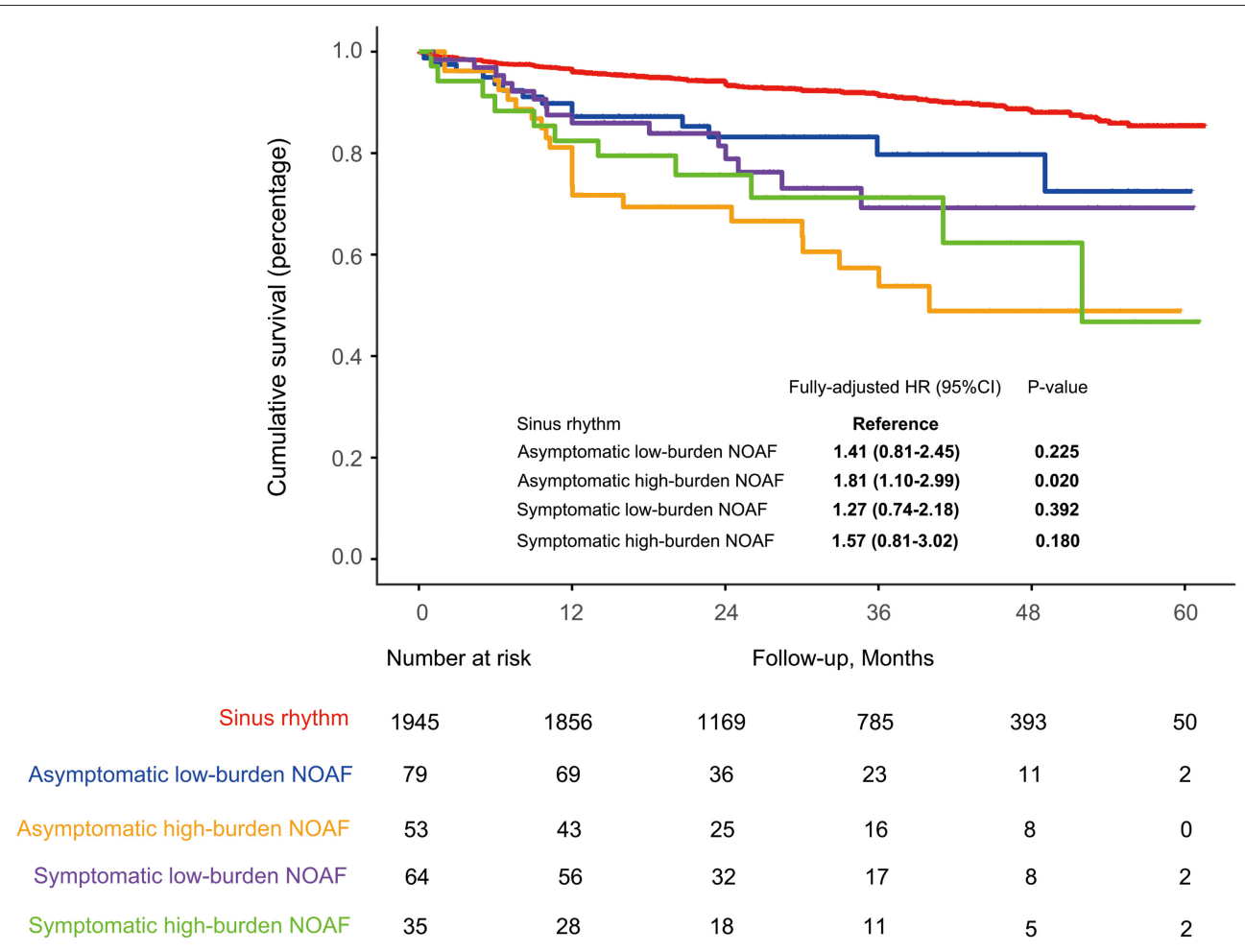

FIGURE 4 | Long-term survival in the whole cohort stratified by the symptom and burden level of NOAF during AMI. HF, heart failure; NOAF, new-onset atrial fibrillation; PS, propensity score; SR, sinus rhythm.

reported in a French registry where nearly $16.0 \%$ of the AMI individuals presented with asymptomatic AF (13). We postulated the difference might be explained as follows: the former study included patients with pre-existing AF who might have received $\beta$-blockers for rate control, thus alleviating the symptoms of AF. Besides, the high usage rate of amiodarone for cardioversion purposes reflects our concerns on the detrimental impact of postMI NOAF, and this could also contribute to the low rate of asymptomatic $\operatorname{AF}(11,12)$.

An important finding of our study was that the impacts of asymptomatic and symptomatic NOAF episodes on shortand long-term survival were divergent. After adjustment for conventional cardiovascular confounders, we showed that symptomatic NOAF was associated with 2-fold increased mortality during hospitalization; in contrast, only the asymptomatic NOAF was significantly related to poor longterm survival (HR: $1.72,95 \% \mathrm{CI}: 1.17-2.53, P=0.005$ ). The robustness of our results was further validated in the PSM and IPTW cohorts. Preceding studies had identified several pivotal risk factors of in-hospital death, such as age, heart rate, SBP, Killip class, etc. $(19,20)$. Given the fact that the majority of aforementioned risk factors presented in the symptomatic NOAF group (Table 1), it was not hard to understand such increased in-hospital mortality.

Debates concerning the prognostic implications of asymptomatic and symptomatic AF still exist in various settings $(4,6,9)$. In a subanalysis of the Atrial Fibrillation
Follow-up Investigation of Rhythm Management (AFFIRM) trial, although the crude mortality was higher in the symptomatic AF group compared with that in the asymptomatic group (27 vs. $19 \%$ ), statistical significance was not achieved after adjusting for a history of coronary artery disease, HF, and LVEF (HR: 1.07, 95\% CI: 0.79-1.46, $P=0.67$ ) (7). By contrast, Boriani et al. demonstrated that asymptomatic AF was significantly associated with increased 1-year mortality as compared with symptomatic AF (9.4 vs. $4.2 \%, P<0.0001)$ (3). This time, we demonstrated that the asymptomatic NOAF was significantly associated with poor long-term survival. Similarly, Stamboul et al. had also reported that when treating patients with SR as the reference, those with asymptomatic AF during AMI were at higher risk of 1-year cardiovascular events even after multivariate adjustment (OR: 2.24, 95\% CI: 1.02-4.93, $P=0.046$ ) (15). Although the exact mechanism was still unclear, we considered this could partially be ascribed to the insufficient clinical concerns for patients without AF symptoms, thus leading to the inappropriate or delayed use of optimal management (4). Besides, Guenancia et al. showed that $\mathrm{AF}$ recurrences were more frequent in patients with symptomatic AF during AMI than in those with asymptomatic $\mathrm{AF}$, which would make the asymptomatic NOAF even more difficult to be detected and treated (21). Our exploratory analysis in which patients with asymptomatic high-burden AF episodes (AF burden $>10.87 \%$ ) were recognized as the highest-risk population underscored the clinical importance of strengthened ECG monitoring and AF burden control, since the 
asymptomatic AF had been determined by Potpara et al. as more likely to progress into a permanent pattern when compared to the symptomatic one (HR: 1.6, 95\% CI: 1.1-2.2, $P=0.009$ ) (8). Technical advances with respect to AF detection, for example, the use of smart device-based photoplethysmography technology (22), may be useful in patients with NOAF during AMI for the long-term AF screening, AF burden evaluation, as well as further clinical decision-making.

In line with prior studies, our results with respect to $\mathrm{HF}$ hospitalization further corroborated the fact that NOAF was an important risk factor of $\mathrm{HF}$ after $\operatorname{AMI}(17,23)$, which was independent of AF symptoms. Interestingly, we found that only the symptomatic NOAF during AMI was significantly associated with an increased risk of ischemic stroke, which was different from previous reports that patients with asymptomatic AF were at high risk of ischemic stroke due to suboptimal anticoagulation $(8,24)$. We assumed it might be explained by the low usage rate of oral anticoagulant (OAC) among post-MI NOAF individuals $(\approx 7.4 \%)$; therefore, OAC treatment would have little impact on the analyzed population. In fact, as reported in the Chinese Acute Myocardial Infarction (CAMI) registry, only 5.1\% of AMI patients concomitant with AF had been prescribed warfarin, and the rate of the combined use of warfarin and dual antiplatelet was even lower $(\approx 1.7 \%)$ (25). Such a striking gap may be due to the careful prescription of OAC after AMI given the risk of intracranial hemorrhage is higher in the Asian population (26). Accordingly, based on the present study, we postulated that patients' clinical profiles could be the dominant factor for the elevated risk of ischemic stroke, as patients with symptomatic NOAF had a relatively higher $\mathrm{CHA}_{2} \mathrm{DS}_{2}$-VASc score compared to those with asymptomatic NOAF ( $3.6 \pm 1.8$ vs. $4.0 \pm 1.8$; Table 1$)$.

\section{Limitations}

The present analysis is retrospective in nature and thus subject to limitations about the uniformity of data collection. However, we performed a manual review of all admission records, rather than rely on coded information to both adjudicate the diagnosis of AMI as well as NOAF ascertainment. Although patients with a documented history of AF had been excluded, we cannot eliminate the possibility of NOAF misclassification as patients with an undiagnosed AF may be included. Because of lacking data on the specific causes of death (e.g., due to HF, stroke, bleeding, etc.), we cannot evaluate the association of the NOAF symptoms with cause-specific mortality. Also, the low rate of oral anticoagulant usage may limit the generalization

\section{REFERENCES}

1. Gladstone DJ, Spring M, Dorian P, Panzov V, Thorpe KE, Hall J, et al. Atrial fibrillation in patients with cryptogenic stroke. N Engl J Med. (2014) 370:2467-77. doi: 10.1056/NEJMoa1311376

2. Sanna T, Diener HC, Passman RS, Di Lazzaro V, Bernstein RA, Morillo CA, et al. Cryptogenic stroke and underlying atrial fibrillation. $N$ Engl J Med. (2014) 370:2478-86. doi: 10.1056/NEJMoa1313600

3. Boriani G, Laroche C, Diemberger I, Fantecchi E, Popescu MI, Rasmussen LH, et al. Asymptomatic atrial fibrillation: clinical correlates, management, of our results to other cohorts. Finally, the number of patients who developed NOAF in this study is limited, and further studies with a larger sample size are highly desirable to confirm our findings.

\section{CONCLUSIONS}

Our results indicated that patients with post-MI symptomatic NOAF were the high-risk population of in-hospital death, and those with asymptomatic NOAF, especially concomitant with a high AF burden, had poor long-term survival. These findings highlight the importance of strengthened management for symptomatic NOAF episodes during the acute phase of AMI and the usefulness of extensive ECG monitoring among patients with asymptomatic NOAF to facilitate AF detection as well as timely initiation of treatment.

\section{DATA AVAILABILITY STATEMENT}

The raw data supporting the conclusions of this article will be made available by the authors, without undue reservation.

\section{ETHICS STATEMENT}

The studies involving human participants were reviewed and approved by The ethics committee of the Shanghai Tenth People's Hospital. The ethics committee waived the requirement of written informed consent for participation.

\section{AUTHOR CONTRIBUTIONS}

All authors listed have made a substantial, direct and intellectual contribution to the work, and approved it for publication.

\section{FUNDING}

This work was supported by the Natural Science Foundation of Shanghai (18ZR1429700) and the National Natural Science Foundation of China (81270193 and 81900385).

\section{SUPPLEMENTARY MATERIAL}

The Supplementary Material for this article can be found online at: https://www.frontiersin.org/articles/10.3389/fcvm. 2021.677695/full\#supplementary-material 
6. Siontis K, Gersh B, Killian J, Noseworthy P, McCabe P, Weston S, et al. Typical, atypical, and asymptomatic presentations of new-onset atrial fibrillation in the community: Characteristics and prognostic implications. Heart Rhythm. (2016) 13:1418-24. doi: 10.1016/j.hrthm.2016.03.003

7. Flaker GC, Belew K, Beckman K, Vidaillet H, Kron J, Safford R, et al. Asymptomatic atrial fibrillation: demographic features and prognostic information from the Atrial Fibrillation Follow-up Investigation of Rhythm Management (AFFIRM) study. Am Heart J. (2005) 149:657-63. doi: 10.1016/j.ahj.2004.06.032

8. Potpara TS, Polovina MM, Marinkovic JM, Lip GY. A comparison of clinical characteristics and long-term prognosis in asymptomatic and symptomatic patients with first-diagnosed atrial fibrillation: the Belgrade Atrial Fibrillation Study. Int J Cardiol. (2013) 168:4744-9. doi: 10.1016/j.ijcard.2013.07.234

9. Rienstra M, Vermond RA, Crijns HJ, Tijssen JG, Van Gelder IC. Asymptomatic persistent atrial fibrillation and outcome: results of the RACE study. Heart Rhythm. (2014) 11:939-45. doi: 10.1016/j.hrthm.2014.03.016

10. Thind M, Holmes D, Badri M, Pieper K, Singh A, Blanco R, et al. Embolic and other adverse outcomes in symptomatic versus asymptomatic patients with atrial fibrillation (from the ORBIT-AF Registry). Am J Cardiol. (2018) 122:1677-83. doi: 10.1016/j.amjcard.2018.07.045

11. Jabre P, Roger VL, Murad MH, Chamberlain AM, Prokop L, Adnet F, et al. Mortality associated with atrial fibrillation in patients with myocardial infarction: a systematic review and meta-analysis. Circulation. (2011) 123:1587-93. doi: 10.1161/circulationaha.110.986661

12. Luo J, Li H, Qin X, Liu B, Zhao J, Maihe G, et al. Increased risk of ischemic stroke associated with new-onset atrial fibrillation complicating acute coronary syndrome: A systematic review and meta-analysis. Int J Cardiol. (2018) 265:125-31. doi: 10.1016/j.ijcard.2018.04.096

13. Stamboul K, Zeller M, Fauchier L, Gudjoncik A, Buffet P, Garnier $\mathrm{F}$, et al. Incidence and prognostic significance of silent atrial fibrillation in acute myocardial infarction. Int $J$ Cardiol. (2014) 174:611-7. doi: 10.1016/j.ijcard.2014.04.158

14. Kirchhof P, Benussi S, Kotecha D, Ahlsson A, Atar D, Casadei B, et al. 2016 ESC Guidelines for the management of atrial fibrillation developed in collaboration with EACTS. Eur Heart J. (2016) 37:2893962. doi: 10.1093/eurheartj/ehw210

15. Stamboul K, Zeller M, Fauchier L, Gudjoncik A, Buffet P, Garnier F, et al. Prognosis of silent atrial fibrillation after acute myocardial infarction at 1-year follow-up. Heart. (2015) 101:864-9. doi: 10.1136/heartjnl-2014-3 07253

16. Luo J, Xu S, Li H, Li Z, Liu B, Qin X, et al. Long-term impact of new-onset atrial fibrillation complicating acute myocardial infarction on heart failure. ESC Heart Fail. (2020) 7:2762-72. doi: 10.1002/ehf2.12872

17. Luo J, Xu S, Li H, Gong M, Li Z, Liu B, et al. Long-term impact of the burden of new-onset atrial fibrillation in patients with acute myocardial infarction: results from the NOAFCAMI-SH registry. Europace. (2021) 23:196-204. doi: 10.1093/europace/euaa234

18. Dilaveris PE, Kennedy HL. Silent atrial fibrillation: epidemiology, diagnosis, and clinical impact. Clin Cardiol. (2017) 40:413-8. doi: 10.1002/clc.22667
19. Granger CB, Goldberg RJ, Dabbous O, Pieper KS, Eagle KA, Cannon CP, et al. Predictors of hospital mortality in the global registry of acute coronary events. Arch Intern Med. (2003) 163:2345-53. doi: 10.1001/archinte.163.19.2345

20. McNamara RL, Kennedy KF, Cohen DJ, Diercks DB, Moscucci M, Ramee S, et al. Predicting in-hospital mortality in patients with acute myocardial infarction. J Am Coll Cardiol. (2016) 68:626-35. doi: 10.1016/j.jacc.2016.05.049

21. Guenancia C, Toucas C, Fauchier L, Stamboul K, Garnier F, Mouhat B, et al. High rate of recurrence at long-term follow-up after new-onset atrial fibrillation during acute myocardial infarction. Europace. (2018) 20:e17988. doi: 10.1093/europace/euy168

22. Guo $\mathrm{Y}$, Wang $\mathrm{H}$, Zhang $\mathrm{H}$, Liu $\mathrm{T}$, Liang $\mathrm{Z}$, Xia $\mathrm{Y}$, et al. Mobile photoplethysmographic technology to detect atrial fibrillation. J Am Coll Cardiol. (2019) 74:2365-75. doi: 10.1016/j.jacc.2019.08.019

23. Asanin M, Perunicic J, Mrdovic I, Matic M, Vujisic-Tesic B, Arandjelovic A, et al. Prognostic significance of new atrial fibrillation and its relation to heart failure following acute myocardial infarction. Eur J Heart Fail. (2005) 7:671-6. doi: 10.1016/j.ejheart.2004.07.018

24. Healey JS, Connolly SJ, Gold MR, Israel CW, Van Gelder IC, Capucci A, et al. Subclinical atrial fibrillation and the risk of stroke. N Engl J Med. (2012) 366:120-9. doi: 10.1056/NEJMoa1105575

25. Dai Y, Yang J, Gao Z, Xu H, Sun Y, Wu Y, et al. Atrial fibrillation in patients hospitalized with acute myocardial infarction: analysis of the china acute myocardial infarction (CAMI) registry. BMC Cardiovasc Disord. (2017) 17:2. doi: 10.1186/s12872-016-0442-9

26. van Asch CJ, Luitse MJ, Rinkel GJ, van der Tweel I, Algra A, Klijn CJ. Incidence, case fatality, and functional outcome of intracerebral haemorrhage over time, according to age, sex, and ethnic origin: a systematic review and meta-analysis. Lancet Neurol. (2010) 9:167-76. doi: $10.1016 /$ s1474-4422(09)70340-0

Conflict of Interest: The authors declare that the research was conducted in the absence of any commercial or financial relationships that could be construed as a potential conflict of interest.

Publisher's Note: All claims expressed in this article are solely those of the authors and do not necessarily represent those of their affiliated organizations, or those of the publisher, the editors and the reviewers. Any product that may be evaluated in this article, or claim that may be made by its manufacturer, is not guaranteed or endorsed by the publisher.

Copyright (C) 2021 Luo, Liu, Li, Xu, Gong, Li, Qin, Shi, Hao, Zhang and Wei. This is an open-access article distributed under the terms of the Creative Commons Attribution License (CC BY). The use, distribution or reproduction in other forums is permitted, provided the original author(s) and the copyright owner(s) are credited and that the original publication in this journal is cited, in accordance with accepted academic practice. No use, distribution or reproduction is permitted which does not comply with these terms. 